\title{
The Incentive to Invest in Environmental-Friendly Technologies: Dynamics Makes a Difference
}

\author{
Davide Dragone* Luca Lambertini* ${ }^{*}$ Arsen Palestini* \\ *Department of Economics, University of Bologna \\ Strada Maggiore 45, 40125 Bologna, Italy \\ fax $+39-051-2092664$ \\ davide.dragone@unibo.it; luca.lambertini@unibo.it; palestini@math.unifi.it \\ $\S$ ENCORE, University of Amsterdam \\ Roeterstraat 11, WB1018 Amsterdam, The Netherlands
}

March 2, 2009 


\begin{abstract}
The established view on oligopolistic competition with environmental externalities has it that, since firms neglect the external effect, their incentive to invest in $R \& D$ for pollution abatement is nil unless they are subject to some form of environmental taxation. We take a dynamic approach to this issue, using a simple differential game to show that the conclusion reached by the static literature is not robust, as the introduction of dynamics shows that firms do invest in R\&D for environmental-friendly technologies throughout the game. Moreover, our setup also illustrates the existence of multiple equilibria, only one of which is identified by the corresponding static game.
\end{abstract}

Keywords: pollution, environmental externality, R\&D, optimal control, differential games

JEL Codes: H23, L13, O31, Q55 


\section{Introduction}

The control of polluting emissions damaging the environment is a hot issue and is receiving an increasing amount of attention in the current literature in the field of environmental economics. Most of the existing contributions investigate the design of optimal Pigouvian taxation aimed at inducing firms to reduce damaging emissions, both in monopoly and oligopoly settings. ${ }^{1} \mathrm{~A}$ related stream of literature examines the incentive for firms to carry out R\&D activities in order to introduce environmental-friendly technologies. This requires the introduction of some form of taxation/subsidy by the policy maker, in order to induce firms to take into account the presence of the externality, that they would clearly neglect otherwise. ${ }^{2}$ A third line of research investigates the optimal design of minimum quality standards and/or profit taxation in vertically differentiated industries affected by environmental externalities. ${ }^{3}$

In the present paper, we take a differential game approach to the investigation of environmentally-oriented $R \& D$ efforts in a dynamic Cournot oligopoly model where there is no tax or subsidy linked to the external effect, in order to show that the main message emerging from the corresponding static version of the same game falls short of telling the whole story of the issue at hand. In particular, we describe a scenario where the stock of pollution increases in proportion to industry output, and each firm may invest in $\mathrm{R} \& \mathrm{D}$ in order to diminish its individual contribution to the emission of pollutants.

We show that the game yields multiple steady state equilibria, one of which corresponding to the static Cournot-Nash equilibrium. The striking

\footnotetext{
${ }^{1}$ See Bergstrom et al. (1987), Karp and Livernois (1994), Bovenberg and de Mooij (1997) and Benchekroun and Long (1998, 2002), inter alia.

${ }^{2}$ To this regard, see Downing and White (1986), Milliman and Prince (1989), Damania (1996) and Poyago-Theotoky (2007), inter alia.

${ }^{3}$ See Lutz et al. (2000) and Lombardini-Riipinen (2005), inter alia.
} 
difference between the static analysis and ours is that we prove that the R\&D efforts of firms is indeed positive at any time during the game, along all of the optimal trajectories leading to any of the three steady states. The static game captures the main feature of one of the steady states we identify, but cannot grasp the essence of what happens along the optimal path to this long run equilibrium. In the remaining two cases, the $R \& D$ effort is positive even in correspondence of the steady state point.

The remainder of the paper is structured as follows. Section 2 briefly outlines the static version of the game. The setup of the dynamic problem is laid out in section 3. The differential game is then illustrated in section 4 . In section 5 we compare the profit and welfare performance of the industry in correspondence of the multiple steady state equilibria. Some concluding remarks are in section 6 .

\section{A summary of the static problem}

As a preliminary step, we revisit the static Cournot game in order to highlight the lack of $R \& D$ incentives to decrease the amount of polluting emissions characterising firms. The market is supplied by $N$ single-product homogeneous-good firms. The market demand function is $p=a-Q$, with $Q=\sum_{i=1}^{N} q_{i}, q_{i}$ being firm $i$ 's output. Technology is the same for all firms alike, and it is summarised by the cost function $C=c q_{i}$. Supplying the final good entails a negative environmental externality $S=\sum_{i=1}^{N} b_{i} q_{i}$, where $b_{i}=\bar{b}-k_{i} \geq 0 ; \bar{b}$ measures the marginal contribution of each firm to the stock of pollutants; $k_{i}$ is the R\&D effort of firm $i$ to decrease its individual amount of pollution, ${ }^{4}$ and it involves a convex cost $\Gamma_{i}=r k_{i}^{2}, r>0$. Consequently,

\footnotetext{
${ }^{4}$ Here we assume firm-specific externalities and $R \& D$ activities, as it appears to be reasonable in examining investments in environmental-friendly technologies. Hence, we rule out the possibility of spillovers in $R \& D$.
} 
firm $i$ 's instantaneous profits are $\pi_{i}=(p-c) q_{i}-\Gamma_{i}$. The present game has a two-stage structure: in the first stage, firms non-cooperatively and simultaneously set their respective $R \& D$ efforts; in the second, they compete à la Cournot-Nash. The solution concept is subgame perfection by backward induction.

The optimal individual output in the second stage is $q^{*}=(a-c) /(N+1)$, whereby the profit function at the first stage reads $\pi_{i}=[(a-c) /(N+1)]^{2}-$ $r k_{i}^{2}$. This clearly entails that $\partial \pi_{i} / \partial k_{i}<0$, and therefore the optimal R\&D investment is nil. On this basis, one has to introduce some form of environmental taxation, no matter whether it is firm-specific or not, to induce firms to take into account the presence of the externality and indeed carry out some R\&D efforts to reduce it. As we shall see in the following sections, this is not necessarily the case if one adopts a properly dynamic approach to this issue.

\section{The dynamic setup}

As in the static model, consider a Cournot oligopoly with $N$ single-product homogeneous-good firms interacting over continuous time $t \in[0, \infty)$. At any time $t$, the demand function is $p(t)=a-Q(t)$, with $Q(t)=\sum_{i=1}^{N} q_{i}(t), q_{i}(t)$ being the instantaneous individual output of firm $i$. All firms use the same productive technology, described by the cost function $C(t)=c q_{i}(t)$. The production of the final output involves a negative environmental externality $S(t)$, evolving according to the following dynamics:

$$
\dot{S}(t)=\sum_{i=1}^{N} b_{i}(t) q_{i}(t)-\delta S(t),
$$

where $\delta>0$ is a constant decay rate the initial condition being $S(0)=S_{0}$. The coefficients $b_{i}(t)$ also evolve over time, driven by the nonnegative R\&D 
effort $k_{i}(t)$ of each firm $i$, whereby

$$
\dot{b}_{i}(t)=b_{i}(t)\left[\eta-k_{i}(t)\right], \eta>0 .
$$

That is, until $k_{i}$ keeps smaller than the threshold value $\eta, b_{i}$ is increasing. The instantaneous cost associated with R\&D activity is $\Gamma_{i}(t)=r k_{i}^{2}(t)$, with $r>$ 0 . Hence, firm $i$ 's instantaneous profits are $\pi_{i}(t)=[p(t)-c] q_{i}(t)-\Gamma_{i}(t)$, and firm $i$ has to set $q_{i}(t)$ and $k_{i}(t)$ so as to maximise

$$
\Pi_{i}=\int_{0}^{\infty}\left\{[p(t)-c] q_{i}(t)-\Gamma_{i}(t)\right\} e^{-\rho t} d t
$$

under the set of constraints given by the state equations (1) and (2). Parameter $\rho>0$ is a constant discount rate common to all firms.

\section{The game}

The solution concept is the open-loop Nash equilibrium. The Hamiltonian of firm $i$ is:

$$
\mathcal{H}_{i}(\cdot)=e^{-\rho t}\left\{[p(t)-c] q_{i}(t)-\Gamma_{i}(t)+\lambda_{i} \dot{S}(t)+\mu_{i i} \dot{b}_{i}(t)+\sum_{j \neq i} \mu_{i j} \dot{b}_{j}(t)\right\}
$$

with the following necessary conditions (FOCs):

$$
\frac{\partial \mathcal{H}_{i}}{\partial q_{i}}=a-c-2 q_{i}(t)-Q_{-i}(t)+\lambda_{i}(t) b_{i}(t)=0
$$

where $Q_{-i}(t)=\sum_{j \neq i} q_{j}(t)$;

$$
\frac{\partial \mathcal{H}_{i}}{\partial k_{i}}=-2 r k_{i}(t)-\mu_{i i}(t) b_{i}(t)=0,
$$

whereas the adjoint equations read as follows:

$$
\dot{\lambda}_{i}(t)=(\rho+\delta) \lambda_{i}(t)
$$




$$
\begin{gathered}
\dot{\mu}_{i i}(t)=\left[\rho-\eta+k_{i}(t)\right] \mu_{i i}(t)-\lambda_{i}(t) q_{i}(t) \\
\dot{\mu}_{i j}(t)=\left[\rho-\eta+k_{j}(t)\right] \mu_{i j}(t)-\lambda_{i}(t) q_{j}(t) .
\end{gathered}
$$

From (5) and (6) one obtains, respectively:

$$
\begin{gathered}
\lambda_{i}(t)=-\frac{a-c-2 q_{i}(t)-Q_{-i}(t)}{b_{i}(t)} \\
\mu_{i i}(t)=-\frac{2 r k_{i}(t)}{b_{i}(t)}
\end{gathered}
$$

while the value of any $\mu_{i j}(t)$ is irrelevant due to additive separability.

Now one can impose symmetry across quantities, costate variables and states:

$$
\begin{aligned}
& q_{i}(t)=q_{j}(t)=q(t), \quad \lambda_{i}(t)=\lambda_{j}(t)=\lambda(t), \\
& \mu_{i i}(t)=\mu_{j j}(t)=\mu(t), \quad b_{i}(t)=b_{j}(t)=b(t)
\end{aligned}
$$

and drop the time argument for brevity. From the FOCs (5) and (6) one also obtains the control equations:

$$
\dot{q}=\frac{\lambda \dot{b}+\dot{\lambda} b}{N+1}, \quad \dot{k}=-\frac{\mu \dot{b}+\dot{\mu} b}{2 r}
$$

which can be rewritten, using (7-8) and (10-11), leading to the following state-control dynamical system:

$$
\begin{aligned}
\dot{S}(t) & =b(t) q(t)-\delta S(t) \\
\dot{b}(t) & =b(t)(\eta-k(t)) \\
\dot{q}(t) & =\frac{[(N+1) q(t)-a+c](\rho+\delta+\eta-k(t))}{N+1} \\
\dot{k}(t) & =\rho k(t)-\frac{q(t)[a-c-(N+1) q(t)]}{2 r}
\end{aligned}
$$

Lemma 1 The stationary points of the system are:

$$
P_{A}=\left(S_{A}=0, b_{A}=0, q_{A}=\frac{a-c}{N+1}, k^{*}=0\right),
$$




$$
\begin{aligned}
& P_{B}=\left(S_{B}=0, b_{B}=0, q_{B}, k_{B}\right), \\
& P_{C}=\left(S_{C}=0, b_{C}=0, q_{C}, k_{C}\right),
\end{aligned}
$$

where

$$
\begin{gathered}
q_{B}=\frac{a-c-\sqrt{(a-c)^{2}-8 r(N+1)(\rho+\delta+\eta) \rho}}{2(N+1)} \\
q_{C}=\frac{a-c+\sqrt{(a-c)^{2}-8 r(N+1)(\rho+\delta+\eta) \rho}}{2(N+1)} \\
k_{B}=k_{C}=\delta+\rho+\eta .
\end{gathered}
$$

Proof. Imposing the stationarity condition $\dot{k}=0$ yields

$$
k(q)=\frac{q[a-c-(N+1) q]}{2 r \rho}
$$

which can be plugged into $\dot{q}=0$ to obtain the following solutions:

$$
q_{A}=\frac{a-c}{N+1} ; q_{B, C}=\frac{a-c \pm \sqrt{(a-c)^{2}-8 r(N+1)(\rho+\delta+\eta) \rho}}{2(N+1)}
$$

with $q_{B, C} \in \mathbb{R}_{+}$for $a-c>\sqrt{8 r(N+1)(\rho+\delta+\eta) \rho}$. By substituting in (17) we have that $k_{B, C}=\delta+\rho+\eta$.

In correspondence of the classical Cournot-Nash optimal quantity $q_{1}$, we have $k_{1}=0$. Equations (13-14) only admit the fixed points $S=0, b=0$.

Lemma $2 P_{A}, P_{B}$ and $P_{C}$ are saddle points of the system.

Proof. The Jacobian matrix of the state-control system reads as:

$$
J=\left(\begin{array}{cccc}
-\delta & N q & N b & 0 \\
0 & \eta-k & 0 & -b \\
0 & 0 & \rho+\delta+\eta-k & -q+\frac{a-c}{N+1} \\
0 & 0 & \frac{1}{2 r}[2(N+1) q-a+c] & \rho
\end{array}\right) .
$$


$J\left(P_{A}\right)$ has the eigenvalues $\lambda_{1}=-\delta<0, \lambda_{2}=\eta>0, \lambda_{3}=\rho+\delta+\eta>0$ and $\lambda_{4}=\rho>0$, subsequently $P_{A}$ is a saddle point.

The analysis of the remaining two equilibria is slightly more difficult: both $J\left(P_{B}\right)$ and $J\left(P_{C}\right)$ admit the negative eigenvalues $\lambda_{1}=-\delta<0$ and $\lambda_{2}=-\rho-\delta<0$, so the stability properties of those two points depend on the roots of the characteristic polynomials of the submatrices:

$$
\left(\begin{array}{cc}
\rho+\delta+\eta-k_{j} & -q_{j}+\frac{a-c}{N+1} \\
\frac{1}{2 r}\left[2(N+1) q_{j}-a+c\right] & \rho
\end{array}\right)
$$

i.e.

$$
p_{j}(\lambda)=\lambda^{2}-\rho \lambda-\frac{1}{2 r}\left(-q_{j}+\frac{a-c}{N+1}\right)\left[2(N+1) q_{j}-a+c\right], \quad j=2,3 .
$$

If $j=2$, the two remaining eigenvalues are complex with real part $\rho / 2>0$, whereas if $j=3$, they are real and at least one of them is positive, hence $P_{B}$ and $P_{C}$ are saddle points too.

Although the equations (13-14) and (15-16) are not decoupled, we can carry out a qualitative analysis of the phase curves of the system.

Given any solution curve $(\bar{q}(t), \bar{k}(t))$ of equations (15-16), we can easily obtain the state trajectories by applying separation of variable and Lagrange's variation of constant methods to (13-14). Considering the vector of initial conditions $b(0)=b_{0}, S(0)=S_{0}$, we have:

$$
\begin{gathered}
b(t)=b_{0} e^{\eta t-\int_{0}^{t} \bar{k}(s) d s} \\
S(t)=\left(S_{0}+b_{0} \int_{0}^{t}\left(e^{(\eta+\delta) s-\int_{0}^{s} \bar{k}(\tau) d \tau}\right) \bar{q}(s) d s\right) e^{-\delta t} .
\end{gathered}
$$

As far as the saddle point equilibrium paths are concerned, we can state the following: 
Proposition 3 In the half-space $k>\eta$, along each equilibrium trajectory of the system close to $P_{B}$ and $P_{C}$ the state variables $S$ and $b$ are monotonically decreasing to 0 .

Proof. The stationary points $P_{B}$ and $P_{C}$ belong to the half-space $k>\eta$. By calculating the eigenvectors of $J\left(P_{B}\right)$ and $J\left(P_{C}\right)$, it is straightforward to see that the stable subspaces $E_{s}\left(P_{B}\right)$ and $E_{s}\left(P_{C}\right)$ are spanned by the vectors of the canonical basis of $\mathbb{R}^{4}:(1,0,0,0)$ and $(0,1,0,0)$, that is the trajectories on the respective stable manifolds are heading towards the equilibrium coordinates $S=0, b=0$.

The economic meaning of the previous results is clear: in correspondence of the two points $P_{B}$ and $P_{C}$ the stock of pollution tends to diminish and finally disappears.

Moreover, the optimal R\&D effort of the representative firm is positive at any time $t$ during the game. Or, put it the other way around, any nonzero value of the co-state variable attached to the dynamics of the individual firm's contribution to the increase of the pollution stock ensures that the firm itself has indeed an incentive to invest in $R \& D$ activities for pollution abatement all along the game.

\section{$5 \quad$ Profit and welfare assessment}

We compare optimal quantities, profits and values of social welfare in the three steady states. The following lemma results from a trivial chain of inequalities:

Lemma 4 For every $a, c, N, r, \rho, \delta, \eta$, we have $q_{A}>q_{C}>q_{B}$.

The profit levels in the three cases are the following:

$$
\pi\left(P_{A}\right)=\frac{(a-c)^{2}}{(N+1)^{2}}
$$




$$
\begin{aligned}
& \pi\left(P_{B}\right)=(a-c) q_{B}-N q_{B}^{2}-r(\rho+\delta+\eta)^{2}, \\
& \pi\left(P_{C}\right)=(a-c) q_{C}-N q_{C}^{2}-r(\rho+\delta+\eta)^{2} .
\end{aligned}
$$

To this regard, one can prove:

Proposition 5 Any $\rho \in[0, \delta+\eta)$ suffices to ensure that $\pi\left(P_{A}\right)>\pi\left(P_{C}\right)>$ $\pi\left(P_{B}\right)$.

The intuitition behind the above result is that $P_{A}$ is characterised by a larger output level (which, per se, would be detrimental for profits) but the corresponding R\&D effort is nil (which in turn is good news for profits), while the remaining two steady states are characterised by lower output levels in combination with positive R\&D efforts.

Now we turn to consumer surplus $C S\left(P_{i}\right), i=A, B, C$, in the three equilibria. Note that, in principle, the definition of consumer surplus would be $C S\left(P_{i}\right)=Q_{i}^{2} / 2-S_{i}$; however, $S_{i}=0$ always in steady state. The resulting ranking is summarised in

Proposition 6 Over the entire admissible range of parameters, we have $C S\left(P_{A}\right)>C S\left(P_{C}\right)>C S\left(P_{B}\right)$.

Finally, we evaluate social welfare $S W\left(P_{i}\right)=N \pi\left(P_{i}\right)+C S\left(P_{i}\right), i=$ $A, B, C$, to obtain:

Proposition 7 Over the entire admissible range of parameters, we have $S W\left(P_{A}\right)>S W\left(P_{C}\right)>S W\left(P_{B}\right)$.

Lemma 4 and Propositions 5-7 also entail:

Corollary 8 Any $\rho \in[0, \delta+\eta)$ suffices to ensure that private and social preferences over the spectrum of steady state equilibria are reciprocally aligned. 
This essentially relies upon the fact that the industry R\&D effort in $P_{A}$ is nil. Of course, one has to keep in mind that the existence of a unanimous ranking over steady states does not guarantee that the preferred one will be attainable, as the trajectory followed by the dynamic system will indeed depend on the vector of initial conditions on state variables.

\section{Concluding remarks}

We have revisited the issue of the incentive for firms to carry out R\&D efforts aimed at introducing environmental-friendly technologies. Contrary to the acquired view establishing that such an incentive is lacking due to the fact that firms fail to internalise the environmental externality, the dynamic approach we have adopted in the foregoing analysis shows that firms do have an R\&D incentive in this direction throughout the game, although it may indeed vanish in one specific steady state, which portrays the equilibrium outcome of the corresponding static game. 


\section{References}

[1] Benchekroun, H. and N.V. Long (1998). Efficiency inducing taxation for polluting oligopolists. Journal of Public Economics, 70, 325-342.

[2] Benchekroun, H. and N.V. Long (2002). On the multiplicity of efficiencyinducing tax rules. Economics Letters, 76, 331-336.

[3] Bergstrom, T., J. Cross and R. Porter (1987). Efficiency-inducing taxation for a monopolistically supplied depletable resource. Journal of Public Economics, 15, 23-32.

[4] Bovenberg, A.L. and R.A. de Mooij (1997). Environmental tax reform and endogenous growth. Journal of Public Economics, 63, 207-237.

[5] Damania, D. (1996). Pollution taxes and pollution abatement in an oligopoly supergame. Journal of Environmental Economics and Management, 30, 323-36.

[6] Downing, P.B. and L.J. White (1986). Innovation in pollution control. Journal of Environmental Economics and Management, 8, 225-71.

[7] Karp, L. and J. Livernois (1994). Using automatic tax changes to control pollution emissions. Journal of Environmental Economics and Management, 27, 38-48.

[8] Lombardini-Riipinen, C. (2005). Optimal tax policy under environmental quality competition. Environmental and Resource Economics, 32, 317-36.

[9] Lutz, S., T.P. Lyon and J.W. Maxwell (2000), Quality leadership when regulatory standards are forthcoming. Journal of Industrial Economics, 48, 331-48. 
[10] Milliman, S.R. and R. Prince (1989). Firm incentives to promote technological change in pollution control. Journal of Environmental Economics and Management, 17, 247-65.

[11] Poyago-Theotoky, J.A. (2007). The organization of R\&D and environmental policy. Journal of Economic Behavior and Organization, 62, 6375 . 